\title{
Modifying a Scientific Flight Control System for Balloon Launched UAV Missions
}

\author{
Marc Schwarzbach, Sven Wlach, Maximilian Laiacker \\ German Aerospace Center DLR \\ Muenchner Str. 20 \\ 82234 Wessling, Germany \\ marc.schwarzbach@dlr.de
}

\begin{abstract}
In this paper we present our work on enabling Balloon launched high altitude UAV Missions for an autopilot system previously used only at lower levels in visual line of sight conditions. One field of our research in the context of flying robotics is focused on high altitude pseudo satellites (HAPS). To gain operational experience in high altitude flying and for system and payload testing, a balloon launched small UAV (sub 10kg) system was designed including building an optimized airframe. Balloon launching was chosen because it offers fast and clearly regulated access to the desired altitudes. Our autopilot system has proven its capabilities in many years of flight experiments with different platforms (helicopter and fixed wing). The main characteristics are modularity and easy use for scientists.
\end{abstract}

On the hardware level the task was to integrate the existing segmented systems of the research autopilot in a compact form factor, with the possible use in larger platforms in mind. The design was driven by the special thermal requirements resulting from flying in stratospheric conditions. In the autopilot software, several mission specific functions had to be added, which only required moderate effort due to the modular system design. Major changes included adding a flight termination manager. A launch routine was developed allowing a safe transition from free-fall to stable horizontal flight in thin air after being dropped from the balloon.

Extensive testing was performed to validate the design. Simulating the mission, including balloon ascend, was used to check the mission software. Thermal and pressure conditions at altitude were replicated in a thermal vacuum chamber with additional sensors applied to identify problems. The simulation and control laws were verified by means of low altitude test flights.

\section{TABle of Contents}

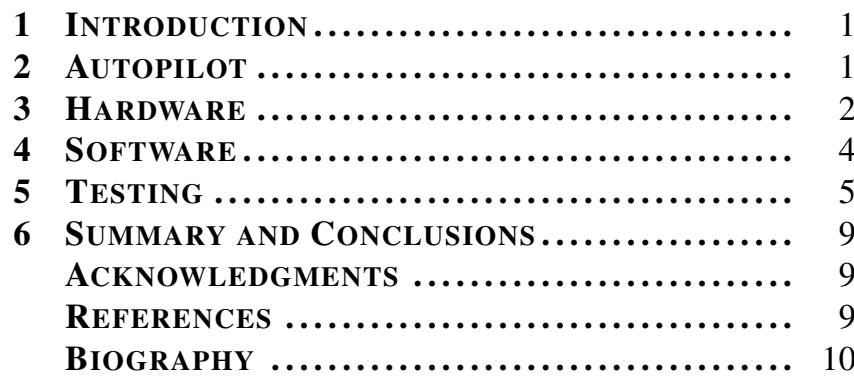

\section{INTRODUCTION}

High altitude platforms have come in focus of research and applications in recent years. They are considered for communication, remote sensing and atmospheric research, to name the most important tasks. The proposed concepts are airplanes and lighter then air vehicles. Most progress

978-1-4799-5380-6/15/\$31.00 (c)2015 IEEE.
Table 1: Technical data of HABLEG

\begin{tabular}{c|c}
\hline \hline Property & Value \\
\hline \hline Wing span & $3 \mathrm{~m}$ \\
Length & $2.4 \mathrm{~m}$ \\
TOM & $7.4 \mathrm{~kg}$ (balloon glider config.) \\
Endurance & 20min. (electric motor); 4h. (glider) \\
\hline \hline
\end{tabular}

has been achieved with solar powered aircraft, beginning with NASA Pathfinder (1993) and Helios (2001) to the two week record flight of Qinetic Zephyr in 2010. Commercial interest has been rising since global players like Facebook and Goolge are also interested in HAPS.

Many challenges arise from the environment the planes have to fly in. Since they have to operate above the clouds to get solar power at all daytime, altitudes of 15 to $20 \mathrm{~km}$ are mandatory. This thin air requires large wings which at the same time have to be very light. Additionally, temperature variations come close to those found in space systems. Airspace regulations today make it hard to find places to perform test flights since controlled airspace has to be crossed.

All these factors lead to the fact that only a few planes were able to perform high altitude test flights to this date. Especially research groups like ours interested in the topic of HAPS need a possibility to perform tests faster and cheaper. We therefore chose the approach to launch a small aircraft from a high altitude balloon. Research in the stratosphere has been conducted using balloons for decades, so regulations and infrastructure is in place for these launches. Other researchers have used similar approaches, for example NASA when simulation a plane to fly on Mars [1].

\section{HABLEG}

The airplane for our experiments, called HABLEG (High Altitude Balloon Launched Experimental Glider), was designed to allow flights from up to $20 \mathrm{~km}$ altitude incorporating avionic systems in development for full scale HAPS. Aerodynamics, structure and thermal systems were constructed, built and tested accordingly. An impression of the size is given in fig. 1. Technical data is found in table 1

\section{Autopilot}

Existing system

Within the flying robots group at DLR (Deutsches Zentrum fuer Luft- und Raumfahrt), the autopilot system has been in continuous development for several years. The key aspect have always been easy adaptability to platforms and usability 


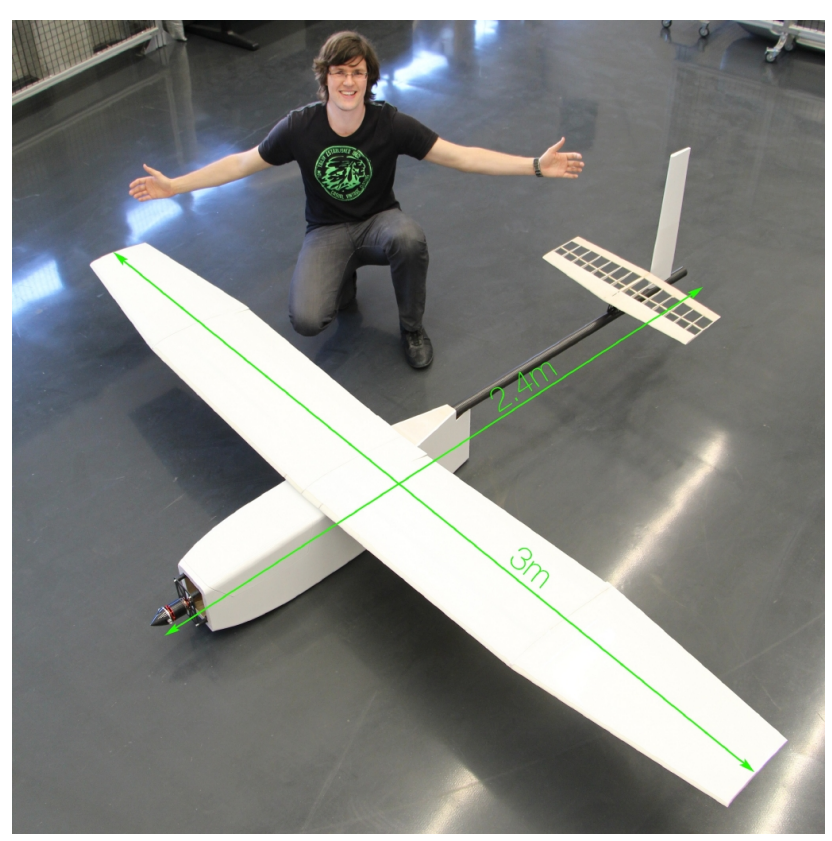

Figure 1: HABLEG assembled

by scientists. An overview of the system highlighting its modularity and scalability is given in [2].

Many projects have used the autopilot in airplanes and helicopters. The tasks range from automatic vision based landing to aerial manipulation using a fully actuated robotic arm mounted on a helicopter [3], [4], [5], [6], [7].

\section{Extension needs}

All projects until now used flying systems in line of sight conditions to the operator and safety pilot. In these conditions, the safety pilot controlling the aircraft within sight like a model aircraft is always a reliable fall back for any kind of failure. Since the desired high altitude mission extends the range to fly by several magnitudes, extensions had to be designed for several aspects of the autopilot system.

The general concept of having a manual control for the safety pilot was kept. The system, however, had to be extended by a long range control link and a live video down-link as pilots view.

The main modifications of the autopilot involved automatic functions for flight execution, safety functions for sensor failure or lost link conditions as well as reliability improvements.

The following sections describe in detail the solutions for specific tasks implemented in hardware and software

\section{HARDWARE}

To achieve maximum flexibility, our existing platforms use the autopilot system built of several hardware modules like main computer, GPS, IMU, which are connected by cables and independent plugs. Changes are thereby quick and platforms can be used for several tasks in short intervals. For the use in HABLEG this modularity was sacrificed for an integrated solution due to thermal and weight constraints. Together with mission specific hardware, namely a temperature

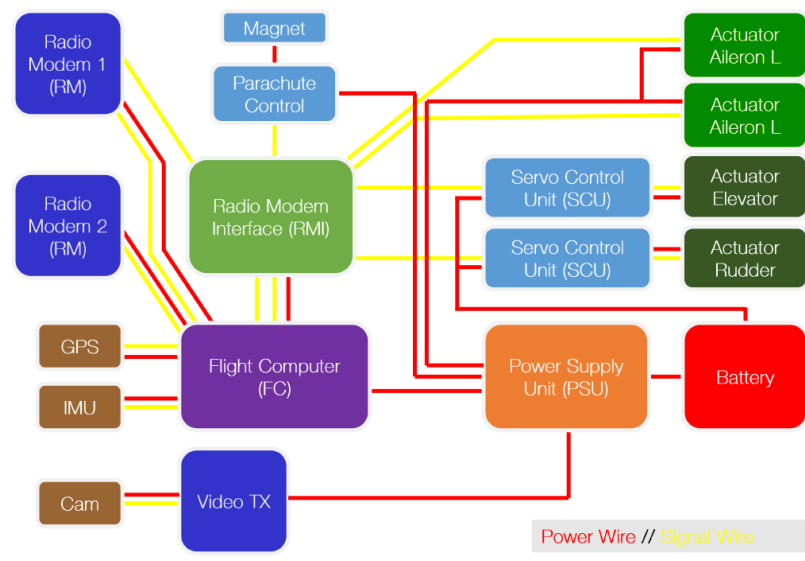

Figure 2: Schematic of wiring between components in the HABLEG system

measurement system and a power supply board, the central avionics hardware was integrated into a single container.

Furthermore, a flight termination system, consisting of a parachute release mechanism and a parachute control board, had to be developed.

\section{Electrical System Overview}

Fig. 2 2 shows a schematic of the components and setup in the HABLEG system. Connections delivering power are marked in red, whereas yellow lines represent signal wires. As it can be seen, the power supply unit (PSU) provides power of different voltages to the flight computer (FC), parachute control board, video transmitter and the actuators of the ailerons. Looking at the signal wires, it can be seen that functions needed for manual control are grouped around the Radio Modem Interface (RMI). It is therefore possible to manually control the aircraft or release the parachute over the primary data link (radio modem 1 in illustration) in case of an autopilot failure. The aileron actuators are conventional servos used for radio-controlled (RC) models and are commanded using pulse-width-modulation (PWM). The actuators for the tailplanes are DLR in-house products driven by dedicated servo control units. The RMI also monitors the FC and has the authority to release the parachute in case of an autopilot malfunction and a simultaneously missing datalink. In a functioning state, the FC receives data from sensors, communicates with the secondary data modem and relays or receives commands over the RMI. It is therefore in full control of all actuators, both data modems and the parachute release.

\section{Avionics Box}

The avionics box is a central part of the HABLEG project. The goal was to design a compact container that includes all major avionics hardware while keeping further use on future stratospheric platforms in mind.

The box is built of carbon fiber plates to provide robustness and shielding against electro-magnetic interference. Fig. 3 shows the front of the avionics box. Here, several connectors interface with the included hardware. On the bottom, four D-sub connectors are responsible for the power and signal lines to the two DLR harmonic drive actuators located in the 


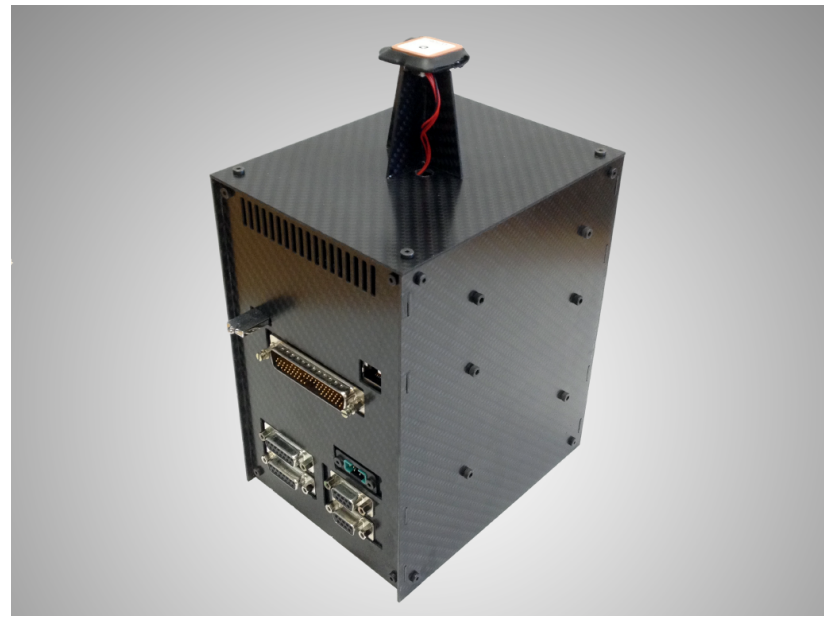

Figure 3: Front of avionics box

payload bay. A 78-pin D-Sub connector provides a single connection to access power rails, serial ports and PWM output channels. With these, all peripheral hardware is powered and communication is achieved. A network port is used for diagnosis of the flight computer on the ground. At the very top end of the front a ventilation grille releases the warm air into the battery bay. Another air outlet is placed on the back of the avionics box, which is especially important during the ascent. Since the glider is oriented nose-down, the air, heated mostly by the CPU and power supply unit, is able to exit over the now overhead located opening in the back plate.

The inner structure is greatly hollowed to save weight and to allow for a free air circulation. Fig. 4 presents a view on the hardware stack, as seen with a removed back plate. By mostly equipping the trays from both sides, additional weight savings were possible. Starting from the top, the first level (1) houses the radio modem interface and the inertial measurement unit, followed by the flight computer on the second level (2). On the same plate as the FC, the power supply unit (3) is mounted. The last tray holds the primary data link modem (4), two servo control units (5) and the foldable antenna of the primary data link (7). The bottom of the box has a slit shaped opening to allow the antenna to fold in during landing or for placing the box on a desk during developing. This slit is insulated with a fitted Styrofoam block (6) to prevent the inflow of cold air. The connectors on the front are also placed on the trays and have no mechanical fixture to the front plate. The three trays are therefore easily removable.

\section{Flight Termination System}

The permanent flight termination system consists of a parachute that reduces the achievable ground distance drastically and slows the aircraft down to moderate impact speeds. A major safety requirement was to design a release mechanism that reliably deploys the parachute, even in case of a complete power loss.

A rope is attached to the airframe's main structure slightly in front of the center of gravity. From here, it runs over the top of the wing to the back of the fuselage and into the parachute bay, where it is tied to the parachute. Thereby the glider hangs relatively horizontal during a descent on parachute.

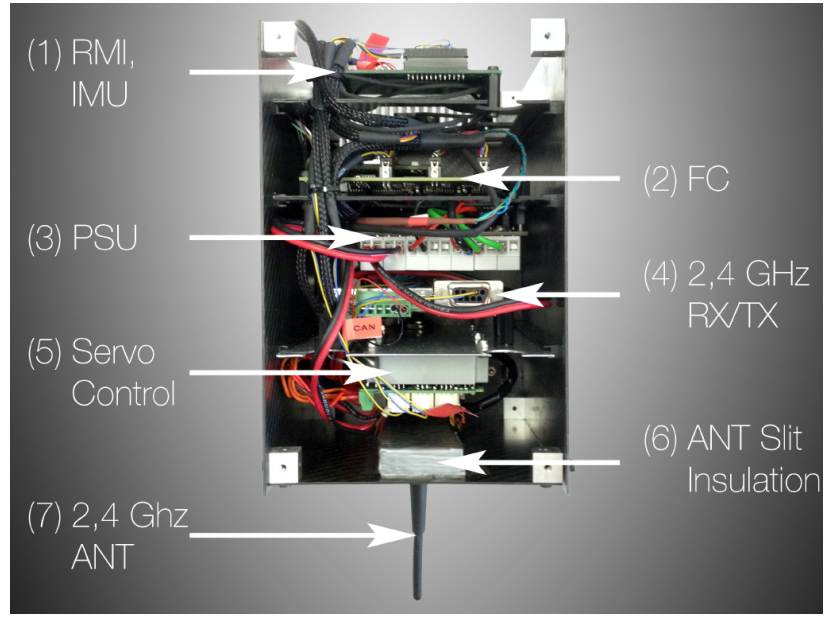

Figure 4: Frontal view on the layers with avionics hardware

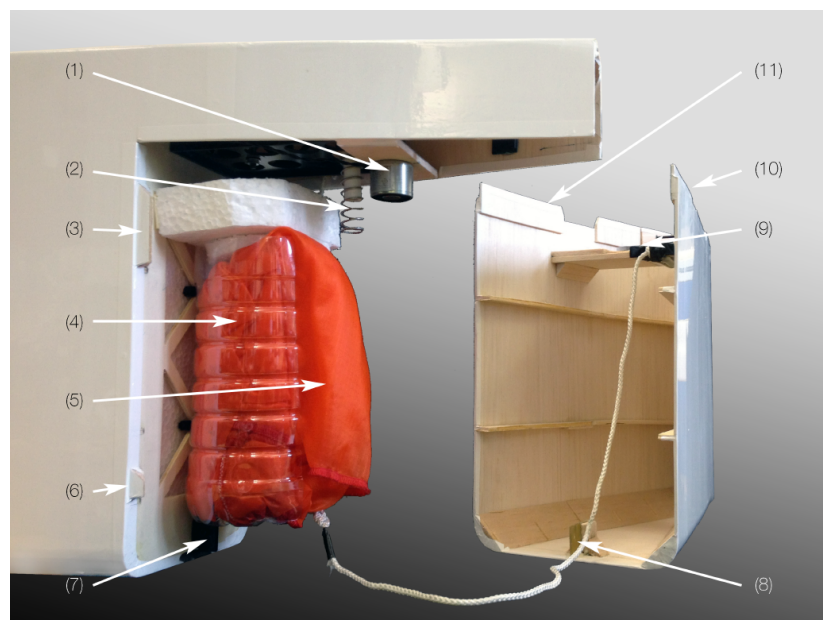

Figure 5: Flight termination system

The attachment point slightly in front of the center of gravity ensures that the tail touches the ground first, thereby reducing impact on the avionics bay.

Fig. 5 shows the inside of the parachute bay with the cover detached. The parachute (5) is folded inside a holder (4). The parachute is attached to the parachute bays cover with a rope. This cover is spring-loaded (2) and kept in place by several guides $(3,6,7,8,10,11)$ and an electro magnet (1). This magnet is controlled by a separate micro-controller (Atmel ATtiny45), located in the payload bay, which receives a live or release signal from the radio modem interface (RMI). If the pulsing live signal from the RMI stops or a release signal is received, the magnet is deactivated. A power loss of the system would as well affect the magnet. Therefore both cases lead to the hood being jettisoned by the spring, which then pulls out the parachute. The concept required a clean alignment of all the guiding parts as well as the magnet and its metal counter plate (9), to ensure a reliable jettison of the hood, while still securing it safely under all the aerodynamic loads during normal operation. To maximize the holding forces, both, magnet and counter plate, were sanded and polished. 


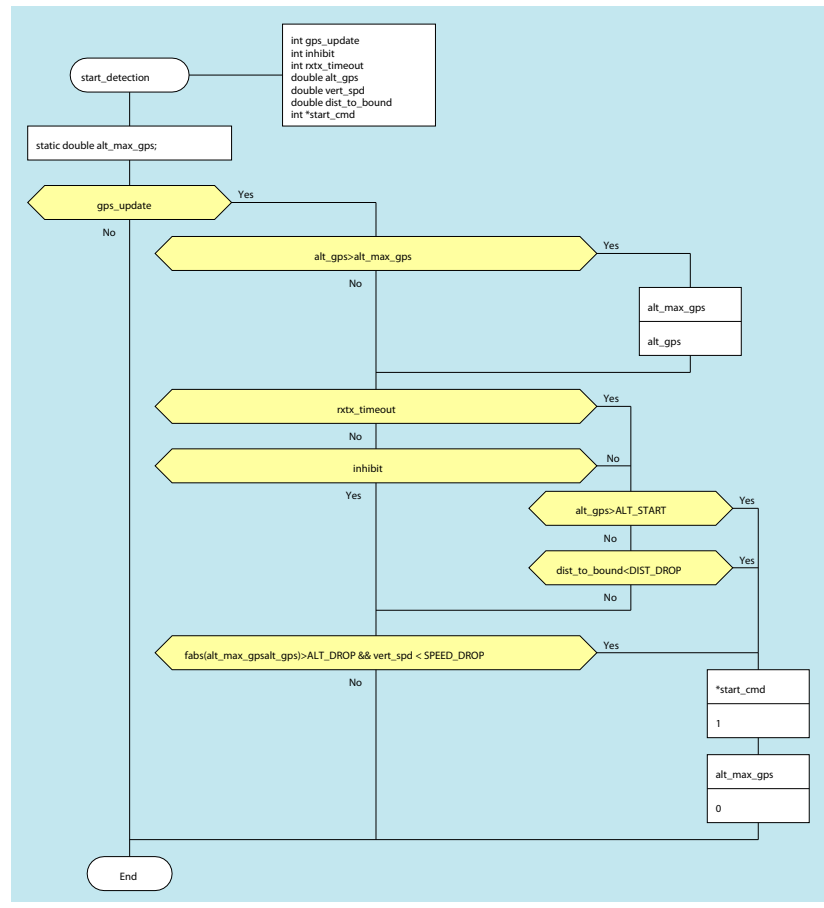

Figure 6: Drakon representation of launch detection code

\section{Software}

The autonomy of the autopilot software had to be increased, especially for possible phases of lost datalink with no possibility of manual intervention. These situations rarely occur in close range testing. Some of the logic implemented is dictated by the safety regulations of the flight test range. Another challenging phase is the release of the plane hanging from the balloon, where automation is most important since it is the point of maximum distance to the ground station and the process needs quick automatic sequencing.

\section{Mission execution}

For programming automatic mission state switching and safety logic our software had to be extended. Like the hardware of our system, the software is modular, so we could add components rather then having to alter existing code. To code these critical functions we are using the DRAKON Editor software originally developed for the Russian space program [8]. It allows to graphically program flow diagrams which are checked for consistency. C-code is generated from the diagrams. The start detection logic is shown in Fig. 6 as an example.

\section{Launch Strategy and Implementation}

The greatest challenge consists in launching the plane in the low density atmosphere. Since the air is so thin, the aircraft needs to fall for a period of time to reach a speed at which flying is possible. Especially in the early drop phase it is possible that an initial rotation, for example due to a kick received upon release might lead to an inescapable flat spin. In fact, prior projects revealed that this is even more problematic than one might think initially. The VEXREDUS project, [9] showed that since the plane is still ascending on the balloon upon release, the inertia carries it a few meters further upwards. Due to the reverse flow, the originally nose-down oriented aircraft pitches up so that it ends in an almost horizontal attitude with zero speed. At these altitudes a conventional aircraft configuration, as it is the case with HABLEG, is then very likely to enter a tail spin or flat spin.

According to calculations, the aircraft needs to fly around $45 \mathrm{~m} / \mathrm{s}$ to achieve level flight at an altitude of $20 \mathrm{~km}$. In order to reach this velocity safely, the plane needs to be stabilized during the drop phase. It was decided to realize this with a small drogue chute attached at the end of the tail boom. The launch sequence looks like this:

- During ascent the glider is suspended on a main rope running from the balloon to a clutch located near the elevator. A drogue chute and its rope are stowed in a jar attached to the main rope close to the aircraft. The drogue chute rope is connected to second clutch. All control surfaces are set to neutral position prior to launch.

- When the launch command is given or balloon burst detected, the primary clutch releases the main rope to the balloon.

- The aircraft now starts free-falling, with a probably unsuitable orientation.

- With increasing vertical speed, the drogue chute starts to develop drag and pulls on the tail of the aircraft, which is thereby aligned nose downwards.

- After a fixed time the autopilot is set to dampen roll and pitch moments.

- By either reaching a predefined speed or after reaching a time limit the second clutch is actuated to release the drogue chute

- The autopilot now gently starts to pull out the glider by obeying a preset vertical acceleration limit while keeping the wings leveled.

- After reaching a preset pitch angle or a time limit, the autopilot changes the flight mode to return home.

Later analysis of NASA video material regarding the ARES Mars aircraft project, [10], indicates that they used a similar technique to stabilize their aircraft during the drop phase, which approves the chosen approach.

\section{Flight Termination Manager Logic}

The following flight termination (FT) modes were defined in order to react in a more differentiated way to a situation at hand:

- FT CSD (Control Surfaces Deflection): This sends the aircraft into a tail spin thereby reducing the ground distance traveling capability to a minimum

- FT Para (Parachute): The glider is equipped with a parachute that enables the rescue of the whole system in case of a failure. The parachute dimension is sufficient to decelerate the aircraft to a vertical speed of around $7 \mathrm{~m} / \mathrm{s}$ at sea level. The parachute hatch is kept closed by an electric magnet, so that even in the case of a total power loss, the chute is ejected.

- FT DC (Drogue Chute): The drogue chute, normally used for stabilization during the early drop phase, can be kept on the glider, instead of cutting it loose after a short period of time. Thereby, it is also possible to reduce the gliding distance in case of a detected malfunction during ascent.

In order to develop a logic that handles flight termination, the airspace is divided into different zones that are defined by radii around a corresponding center point, as illustrated in Fig. 7 .

- Zone A: The landing zone. The glider will head for this 


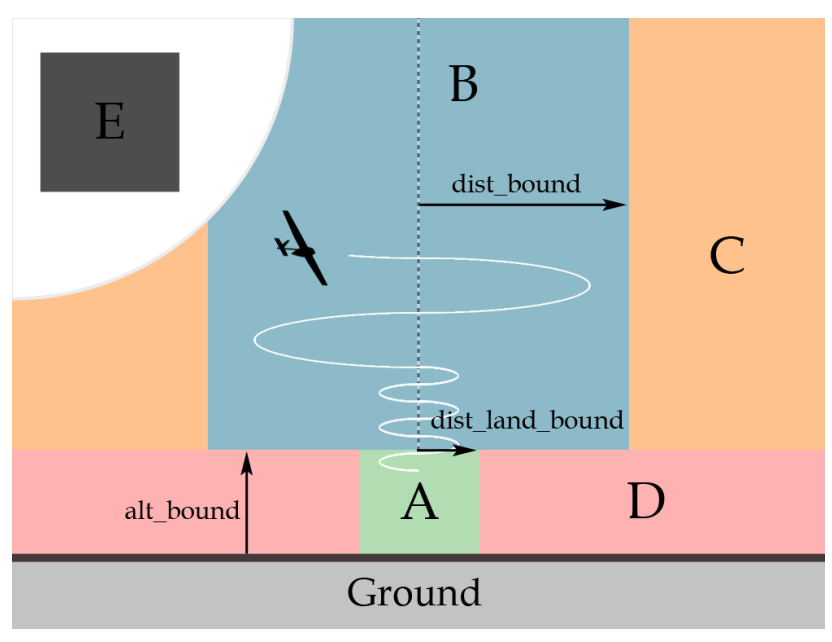

Figure 7: Flying zones

zone during descend. This is the only zone in which it is possible to approach the ground without causing a flight termination on parachute (FT para). It is defined by a radius around the landing location and reaches from ground to an altitude of $1000 \mathrm{~m}$.

- Zone B: This is the regular flying zone in which the ascent on the balloon, drop, experiment phase and fly home procedure will take place. It can be defined by multiple, overlapping circles with different center points.

- Zone C: This is a no-fly zone that will, after a certain amount of time, result in a reversible flight termination by control surface deflection (FT CSD) in case of a breach.

- Zone D: This is also a no-fly zone, but in contrast to Zone $\mathrm{C}$ it will directly cause a permanent flight termination on parachute (FT para)

- Zone E: This is a virtual zone with estimated or unknown position in case of a GPS failure.

The final logic for flight termination is implemented as follows:

The program loop starts by checking the data link connectivity. When passing a time limit a flag for lost connection is set.

The program continues downwards the decision tree by checking if the GPS has a valid lock. If this is the case, but there is a data link loss, it will change the active flight mode to return-to-home, which navigates the glider towards the landing site. The advantage is that in case the aircraft is currently executing a flight test program and loses radio contact it flies towards the base, where a link might be reestablished.

After that, the zone in which the aircraft flies is determined. If it is above the altitude boundary and within the boundaries of zone B, nothing happens. If it is however outside B, the control surfaces of the glider are deflected to send it into a spin (FT spin). Since the loop is restarted from here on, the glider can still be recovered, if the circumstances change, for example by controlling the glider manually.

The just explained behavior is only possible with a valid GPS fix. In case of a GPS loss, the plane has an unknown or only estimated position, which is depicted in Fig. 7 as a virtual zone E.

If there is a healthy data link, the flight computer is set to return home and uses the last known home heading, which is always calculated in case of a valid GPS fix, independent of the active flight mode.

If additionally the data link is lost, the autopilot is set to a GPS and radio signal lost mode. This mode banks the aircraft slowly from left and right, in order to try to reestablish a radio link. However, if that fails and the data link timeout hits the time limit, the logic terminates the flight immediately by deploying the parachute (FT para).

On top of the just explained logic that runs in the flight computer entirely, there is a surveillance logic running on a separate micro-controller, the radio modem interface (RMI) that checks if the flight computer is functional. If this is not the case and there is no data link connection on the primary data link that would allow for manual control, the parachute is deployed. If the RMI stops working, the parachute control board notices the absence of the live signal and releases the parachute.

The last reason for a flight termination is a failure in the power supply of the system. In case of a power loss, the electric magnet stops working as well, thereby releasing the parachute instantly.

\section{TESTING}

In order to validate the design and qualify the system for stratospheric flight, numerous tests were conducted. This included simple functional testing, like the functionality of the release mechanism under load, tests to verify calculated properties, like the drag of the parachute and tests to identify and fix any problems, like RF-range and -interference testing as well as thermal vacuum chamber testing of subsystems. Furthermore, flight tests were conducted to analyze the flying qualities of the aircraft as well as to tune the autopilots control loops. Finally, a partial hardware-in-the-loop simulation was used to test navigational logic, mission scenarios and to perform team training.

\section{RF-Communication Range}

It was estimated that a telemetry range of at least $30 \mathrm{~km}$, corresponding to an altitude and ground distance of about $20 \mathrm{~km}$, should be sufficient for the mission. To simulate a flying aircraft, a test system was placed on a mountaintop whereas the ground station equipment was set up on a lower location with line of sight to the mountain. The flying part of the test setup consisted of the engineering model of the avionic box, batteries, radio modems and servos as pictured in Fig. 9 and was brought by chairlift to the top of the Blomberg, a mountain at the rim of the Alps. Simultaneously, a ground station was setup as seen in Fig. 8 with a $2,4 \mathrm{Ghz}$ dish and an $868 \mathrm{Mhz}$ Yagi-Uda antenna. It is to be noted that no pan-tilt mechanism was used during the test and the additional $5,8 G h z$ meshed dish was unrelated to the experiment. During this test, a ground distance of $28.9 \mathrm{~km}$ was covered with an altitude difference of $460 \mathrm{~m}$. The two data links consisting of a $2,4 G h z$ modem as primary data link and a $868 \mathrm{Mhz}$ Xbee Pro as backup link, were both able to establish a connection. The primary data link reached a data rate sufficient to control the attached servos fluently, parallel to the normal telemetry load. In order to simulate 


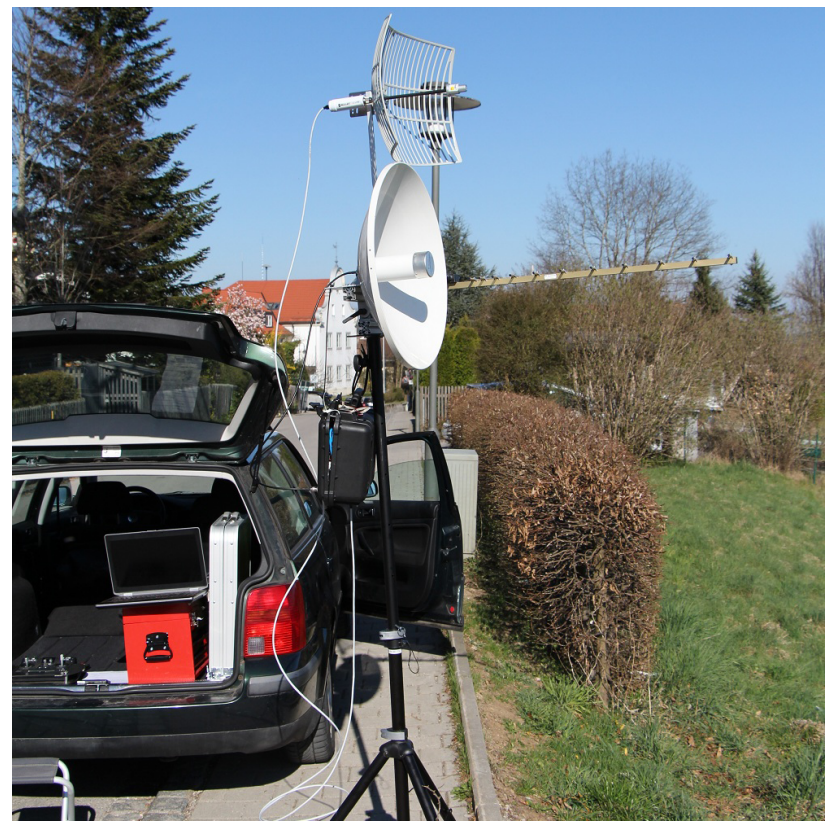

Figure 8: Ground segment setup

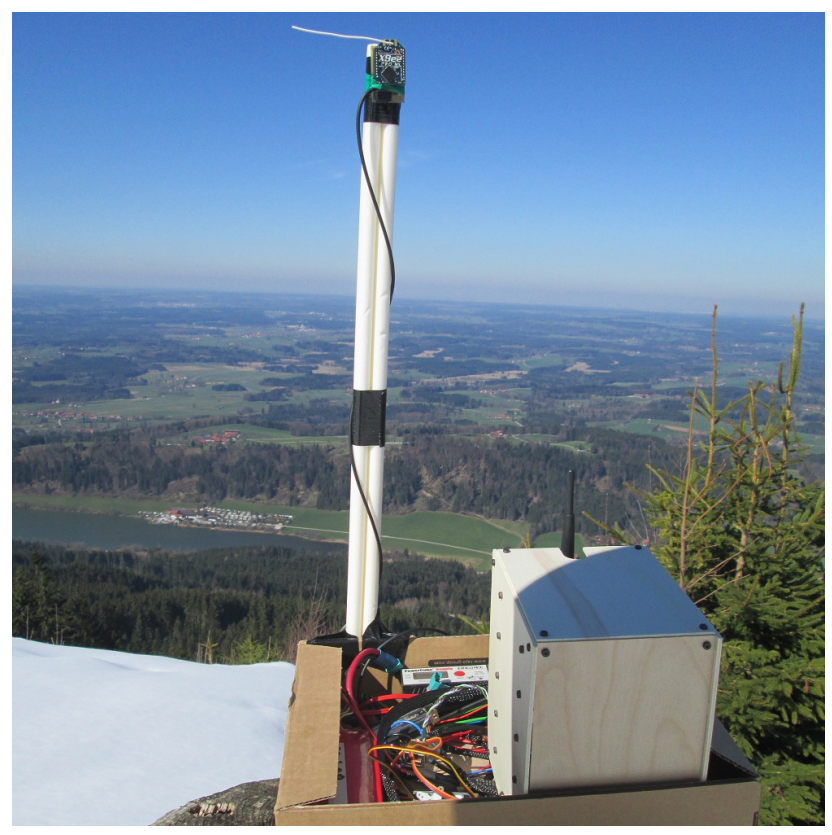

Figure 9: Flying segment setup

the aircrafts movements, the flying end of the setup was tilted in various directions. Here, only a bank angle of over $60^{\circ}$ resulted in a higher packet loss rate and therefore in a stuttering servo reaction to manual control input. It was concluded that even at this distance it would be possible to fly the aircraft manually. Under all conditions the $868 \mathrm{Mhz}$ link showed a solid connection, which was an expected behavior, since the same setup of modem and antenna bridged a distance of $185 \mathrm{~km}$ during a high altitude balloon mission, as described by Kuhn and Wlach, [11]. Though the data rate is not high enough for manual control, the secondary link suits its function as a reliable backup link to relay basic control commands and essential aircraft telemetry.

\section{RF-Interference}

The RF range test already revealed that the present avionic setup does not interfere with the radio transmission. However, it was observed that cell phone calls close to the flying setup interfere with the capability of the system to receive commands from the ground station, which is not a problem for the mission. Nevertheless, if a GSM GPS tracker is to be integrated into the aircraft, further tests are necessary, since such a device sends positional data as SMS over the cellular network.

A further discovery was that the GPS receiver had to be moved further away from the flight computer to obtain a reliable satellite fix. The CPU emits RF signals on the L1 band $(1575.4 \mathrm{Mhz})$ thereby preventing a good GPS lock. The GPS receiver was therefore placed on a stand above and outside the avionics box to gain distance, whereas the carbon shell of the final avionics box design shields electro-magnetic emissions.

In order to verify the functionality of the system under the influence of the video transmitter, a high power system (10W) was borrowed from MORABA. Only when placing the transmitter in a distance of under $0.5 \mathrm{~m}$, the radio links lost the connection. The designated video transmitter only has an output power of $0.5 \mathrm{~W}$, which is less than a third of the tested output power. The test therefore confirmed that the proposed placement of the video transmitter antenna on the outer edge of the center wing, resulting in a distance of over $0.6 \mathrm{~m}$ to the primary and $1.7 \mathrm{~m}$ to the backup data link antenna, is adequate.

Furthermore, since regulations of the rocket base ESRANGE required an aircraft transponder to be flown on the balloon flight train, another interference test was executed directly on site. Though the transponder worked with a pulse transmitting power of $250 \mathrm{~W}$ no adverse effects were noticed.

\section{Thermal Vacuum Chamber}

Flying in stratospheric conditions holds two opposing problems. On the one hand, the cold surrounding air of under $-60^{\circ}$ is a problem for most electronics, especially for components like batteries or the IMU. On the other hand, the low density of the air decreases the rate at which heat can be conductively transferred away from components. Heat producing elements, like a CPU for example, might therefore be prone to overheat. This problem can be mitigated by using heat sinks and sufficient ventilation. The idea of the design is to use the heat produced by hot components to moderate the temperatures in areas like the battery and the payload bay without the need for additional heaters. This had to be validated by a test scenario replicating the environmental conditions. This task can be partly achieved with the thermal vacuum chamber of the IRS. For the test, the temperatures of the following places are of special interest:

\footnotetext{
- Avionics Box (IMU, CPU, Servo Control, PSU)

- Battery Bay

- Battery

- Payload Bay

- Aileron Servo

- Parachute Magnet

- Video Transmitter

- Outside temperature
} 


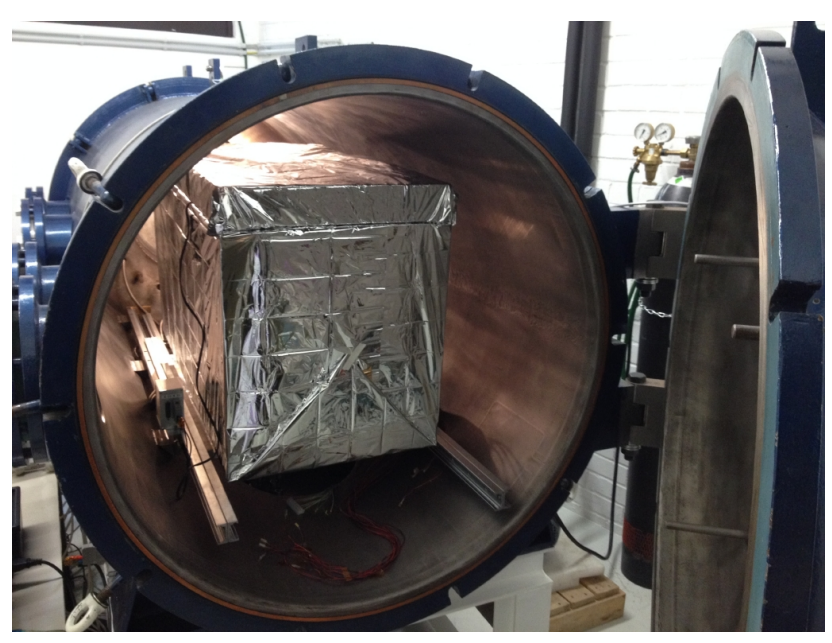

Figure 10: Insulation box inside the thermal vacuum chamber of the IRS

- Various points inside the insulation box

- Cooling plate

- Outside the insulation box

Most of these devices were equipped with temperature sensors that are directly read by a circuit on the custom designed power supply board and relayed to the flight computer, whereas others were instrumented with temperature sensors of the thermal vacuum chamber. To verify the readouts of the on board sensors, some of the measurement points were instrumented in parallel with the calibrated sensors of the chamber. The chamber is of cylindrical shape with a length of $2 \mathrm{~m}$ and a diameter of $1 \mathrm{~m}$. Because of these dimensional restraints, the fuselage was to be tested without the tail boom and the center wing was placed parallel to the fuselage. Another problem is that the chamber was built for subsystem tests. It is therefore not completely cooled, but it has instead a cooling plate that offers an interface temperature necessary to test satellite components. The size of the plate is $400 \mathrm{~mm}$ by $500 \mathrm{~mm}$. Consequently, the goal was to build an insulating box with an interface towards the cooling plate and a way to distribute the coldness evenly. Fig. 10 shows this box inside the chamber, sitting on top of a sliding table with the cooling plate. The box was built from $5 \mathrm{~cm}$ thick Styrofoam plates and covered in space blankets to shield it from thermal radiation of the surrounding chamber. To improve heat transfer to the cooling plate, several heat sinks were scattered across its surface. A fan is arranged in a way that it blows air onto the cooling plate, thereby enforcing circulation. Fig. 11 shows how the fuselage is placed in the box. Since the wing is too large for the box, it was decided to place it outside, thereby only testing it under low pressure conditions.

Fig. 12 plots the temperature development at several test points during the chamber test. The plot is generated with data from the chamber sensors. For the test the pressure was slowly lowered to about $60 \mathrm{mbar}$ over a time span of $10 \mathrm{~min}$. This corresponds to an ISA altitude of 19 to $20 \mathrm{~km}$. In general, all the systems performed well. The temperature in the avionics box was with a maximum of $38^{\circ} \mathrm{C}$ at the IMU and of $66^{\circ} \mathrm{C}$ at the CPU (not in diagram) relatively warm, but not critical. An interesting behavior was discovered in the curve of one of the $12 \mathrm{~V}$ DC/DC converters, located on the power supply board inside the avionics box. Here, at some

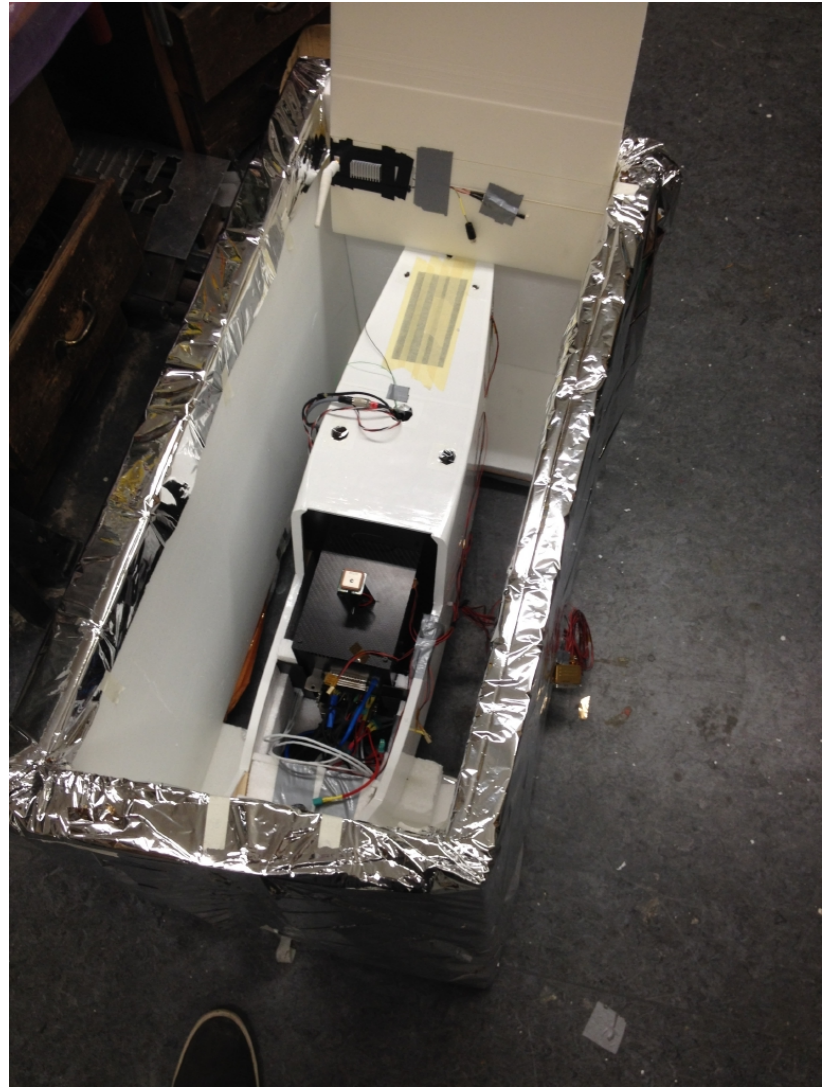

Figure 11: Fuselage with extra temperature sensors and video transmitter test setup

point a spike in the temperature profile appears and reappears after some time. The sudden increase in temperature points to a significantly risen demand on this power rail. The periodicity suggested that it was a controlled event. After some investigation, the source was found within the airdata measurement electronics, which have an integrated heating element that activates as temperatures drop. The system could be adapted to work without the need of the heater.

Another focus was the temperature development of the video transmitter. A first result was that at sea level conditions and without air speed, the transmitter heats up to its operational limits. Therefore, it was decided to use an external fan during longer periods of ground handling. On the other hand, turning on the fan facing the cooling plate resulted in a major temperature drop. Whereas the transmitter itself is located inside the foam core, its heat sink sticks out of the wing's bottom surface and is thereby subjected directly to the cold environment. The great temperature drop, as consequence of enforced convection, led to adding a flow shielding in front of the heat sink in flight direction. This has almost no effect during the low speed ascent, but creates a wake of low speed reversed flow at the heat sink during the flight. By this measure the heat sink can divert enough heat during the low speed ascent, but will not cool the transmitter below a critical level during the flight.

\section{Simulation}

Our modular scalable system for operating and testing of UAVs [2] was also used to implement the simulation. The goal was to simulate all flight phases and failure modes so 


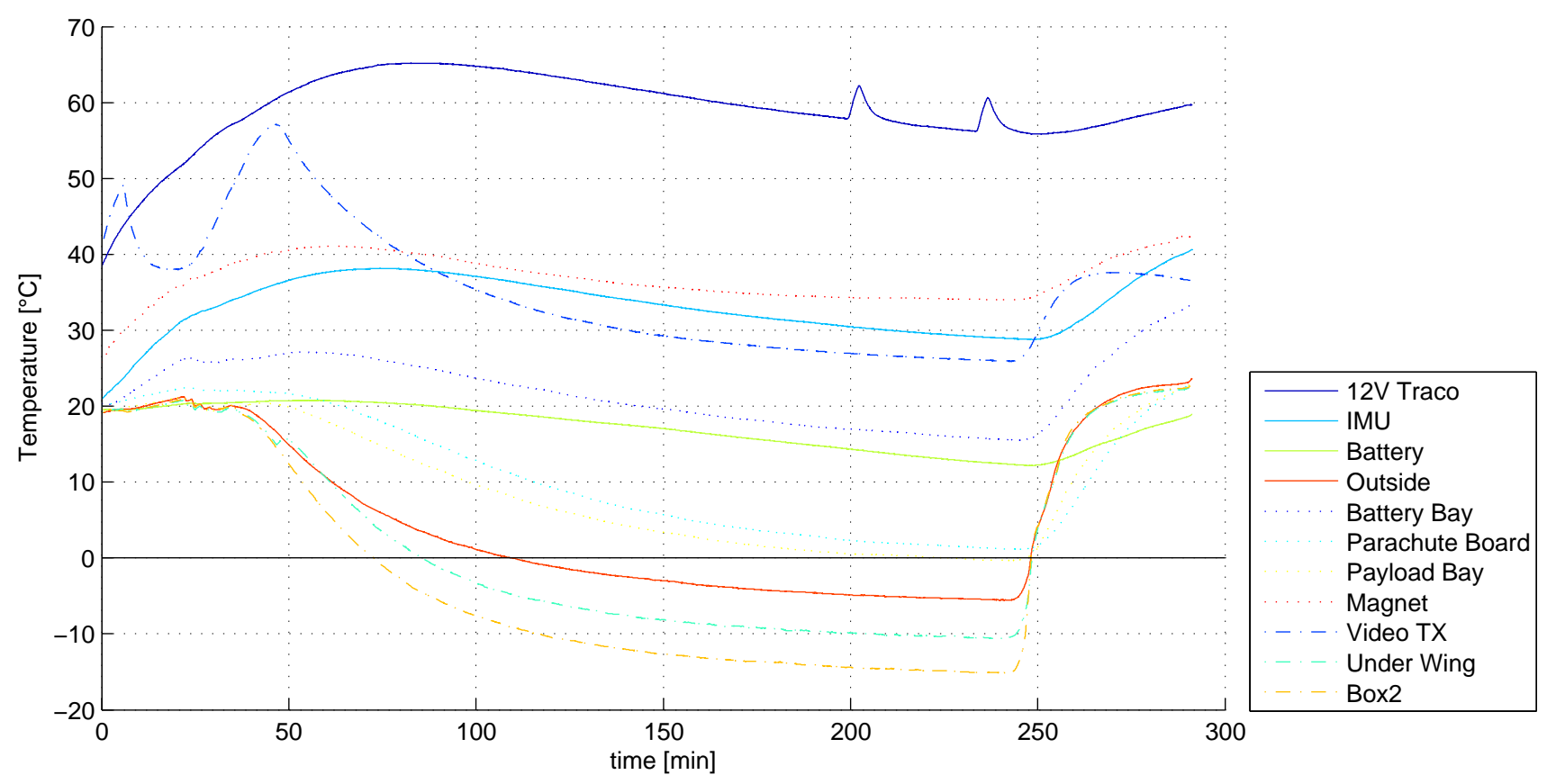

Figure 12: Temperature development during thermal vacuum chamber test

that every part of the mission software can be tested. The simulation process is running on the target system simulating the aircraft reaction to pilot, operator and autopilot commands in real time. While the simulation is running, the processes for handling sensor data are stopped and the simulated model provides this data to the system. The modules that handle the actuator control, communication, logging and flight termination are not changed for the simulation. During the simulation the control surfaces are moving, the operator interface is the same than it would be during a real flight and the safety pilot can also control the plane manually using the remote control as he could during a real flight. The antenna tracking will also move according to the simulated airplane position.

The partial hardware-in-the-loop simulation is able to simulate the HABLEG mission, including the ascent on a balloon. This was used to test the flight termination logic, the launch routine and the reaction to various failure modes, like data link or GPS loss. Wind effects during balloon ascent and unpowered descent can also be studied. It also allowed us to train for the mission and develop operation procedures.

\section{Flight testing}

To perform low altitude flight testing, an aircraft nose with an electric engine, which provides $3.9 \mathrm{~kg}$ using a lithium polymer battery was used. This concept allows for testing independent of a balloon or other means of e.g. towing the aircraft to altitude.

The maiden flight was a purely manual flight using basic $\mathrm{RC}$ equipment. Components of the later planned avionics system like the autopilot, data links or even the harmonic drive servos were not installed on this flight. The aircraft was hand launched, as pictured in Fig. 14. The purpose of this flight experiment was to test general flight characteristics like control surface effectiveness, static stability and stall

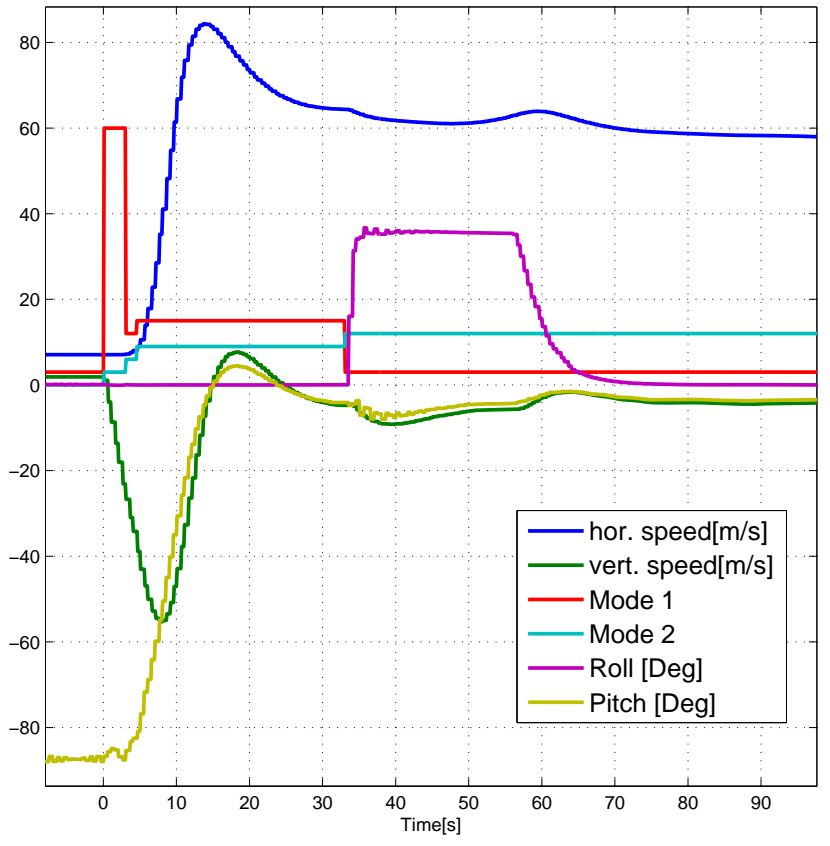

Figure 13: Simulated balloon launch phases, velocities and angles

behavior. The aircraft needed only minor trimming and showed good elevator and aileron reaction. Rudder inputs had a strong but well controllable effect due to the relatively large sized vertical stabilizer. During aerodynamic design, a large tail volume was chosen to provide enough directional stability despite the destabilizing effect of the large fuselage body. This proved to be a correct decision, since the plane showed 


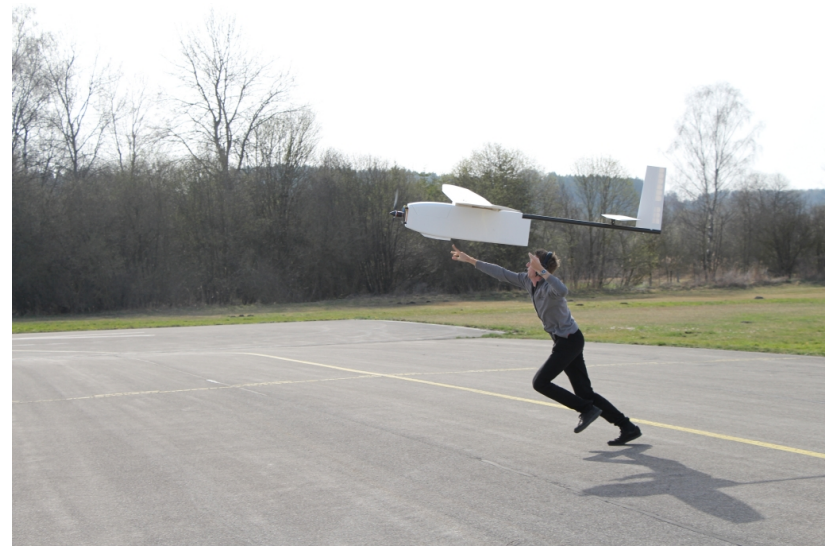

Figure 14: Flight testing HABLEG with electric propulsion

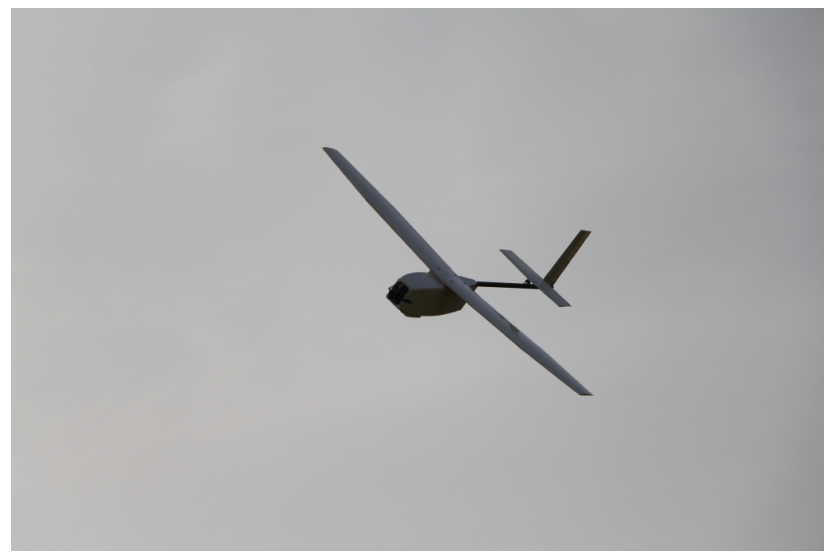

Figure 15: HALEG in flight

a well dampened yaw behavior. Fig. 15 shows HABLEG in flight.

Static stability was tested by suddenly shutting down the electric engine during a slight ascending flight path. The aircraft reacted by gently pitching nose down, accelerating and settling at horizontal flight. This and several dive tests showed a well balanced reaction to disturbances or deviation in speed which confirms a proper static stability as a result of correct CG position and horizontal stabilizer setting.

Finally, stall tests were made while gliding without engine power. The elevator was set at a position that led to a continuous decrease in velocity. At a certain point the glider pitched nose down and accelerated again, which is the intended behavior. Only by then pulling the elevator to maximum deflection, a spiral spin was induced, that was ended instantaneously by returning all control surface to neutral. The stall behavior is therefore docile and well controllable.

The second test flight incorporated the complete set of avionics hardware. Its goal was to tune autopilot control parameters and to test flight routines, like waypoint navigation. Especially the correct behavior of the autopilot in gliding conditions was tested. All the tests were executed successfully and the HABLEG system was therefore declared flight ready.

\section{SumMARY AND CONCLUSIONS}

We have demonstrated that a high altitude balloon launched glider system is feasible to be developed form a short range base system. Several fields of improvement needs were identified and solved. It was very helpful to be able to test subsystems separately like demonstrated by low altitude test flying the airframe with an electric motor. With our sophisticated simulation, guidance and safety software could be developed and tested and team training was possible in a realistic way which made the project possible in the given time and resource constrains. A high altitude flight campaign was performed with only the wind preventing a launch.

With the growing interest in HAPS platforms it is mandatory to push knowledge regarding this technology. Currently solar airplane platforms capable of high altitude long endurance flight are too complex for short term research whereas balloon gondola payloads are hard to control, especially for recovery. Our strategy is to make an intermediate step of combining proven UAV technology with traditional weather balloon experiments to form a system less complex and expensive, while still offering the capability to develop and test numerous aspects needed for large platforms. Such a system could also be used for applications like remote sensing or atmospheric measurements.

\section{ACKNOWLEDGMENTS}

The authors thank SSC and DLR Moraba for supporting this project.

\section{REFERENCES}

[1] J.-C. Lede, R. Parks, and M. Croom, "High altitude drop testing in mars relevant conditions for the ares mars scout mission," in 2nd AIAA "Unmanned Unlimited" Conf. and Workshop, September 2003.

[2] M. Laiacker, A. Klöckner, K. Kondak, M. Schwarzbach, G. Looye, D. Sommer, and I. Kossyk, "Modular scalable system for operation and testing of uavs," in American Control Conference, 2013.

[3] M. Bernard and K. Kondak, "Generic slung load transportation system using small size helicopters," in ICRA. IEEE, 2009, pp. 3258-3264.

[4] M. Schwarzbach, K. Kondak, M. Laiacker, C. Shih, and P. Marron, "Helicopter uav systems for in situ measurements and sensor placement," in Geoscience and Remote Sensing Symposium (IGARSS), 2012 IEEE International, July 2012, pp. 4766-4769.

[5] F. Huber, K. Kondak, K. Krieger, D. Sommer, M. Schwarzbach, M. Laiacker, I. Kossyk, S. Parusel, S. Haddadin, and A. Albu-Schaffer, "First analysis and experiments in aerial manipulation using fully actuated redundant robot arm," in Intelligent Robots and Systems (IROS), 2013 IEEE/RSJ International Conference on. IEEE, 2013, pp. 3452-3457.

[6] K. Kondak, K. Krieger, A. Albu-Schaeffer, M. Schwarzbach, M. Laiacker, I. Maza, A. RodriguezCastano, and A. Ollero, "Closed-loop behavior of an autonomous helicopter equipped with a robotic arm for aerial manipulation tasks," Int J Adv Robotic Sy, vol. 10, no. 145, 2013.

[7] M. Schwarzbach, M. Laiacker, M. Mulero-Pazmany, 
and K. Kondak, "Remote water sampling using flying robots," in Unmanned Aircraft Systems (ICUAS), 2014 International Conference on, May 2014, pp. 72-76.

[8] S. Mitkin, "Drakon : The human revolution in understanding programs." 2011, http://drakoneditor.sourceforge.net/DRAKON.pdf.

[9] D. Steenari, T. Kuhn, and S. Wlach, "Vexredus: A student high altitude glider project to demonstrate the capabilities of a blended wing body concept," in Proc. 21st ESA Symposium on European Rocket \& Balloon Programmes and Related Research, Thun, Switzerland (ESA SP-721, October 2013).

[10] ARES Mars Eagle high altitude drop test, Last accessed: 2014-09-18. [Online]. Available: http:// marsairplane.larc.nasa.gov/graphics/drop.320x240.mov

[11] T. Kuhn and S. Wlach, "Student experiment documentation - vexredus," 2013.

\section{BIOGRAPHY}

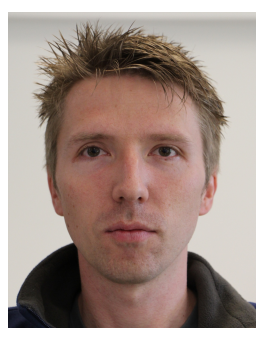

Marc Schwarzbach received his diploma in aerospace engineering from University of Stuttgart in 2005 and a Ph.D. in engineering in 2012. He is currently a member of the Flying Robots research group at the Robotics and Mechatronics Center of the German Aerospace Center DLR. His current research is focused on flying robotics both in the high altitude and close to ground domain.

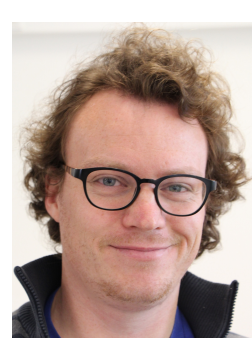

Maximilian Laiacker received his diploma in computer science from $T U$ Berlin in 2009. He is currently a member of the Flying Robots research group at the Robotics and Mechatronics Center of the German Aerospace Center DLR. His main research interests are vision based state estimation, collision avoidance and sensor fusion for flying robots.

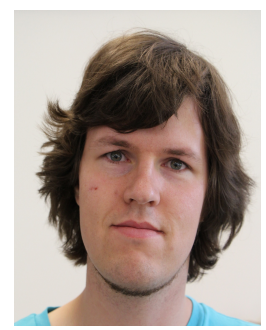

Sven Wlach received his diploma in aerospace engineering from University of Stuttgart in 2014. He is currently a member of the Flying Robots research group at the Robotics and Mechatronics Center of the German Aerospace Center DLR. His current research is focused on High Altitude Pseudo-Satellites (HAPS). 